\title{
Surgical Management of Pyothorax: A Series of 172 Cases
}

\author{
Harmouchi Hicham ${ }^{*}$, Rabiou Sani1, Issoufou Ibrahim¹, Sidibe Kassim², Belliraj Layla1, \\ Ammor Fatimazahra1, Lakranbi Marouane1, Ouadnouni Yassine1,3, Smahi Mohammed ${ }^{1,3}$ \\ ${ }^{1}$ Department of Thoracic Surgery, CHU Hassan II of Fez-Morocco, Fez, Morocco \\ ${ }^{2}$ Department of Imagery CHU Hassan II of Fez-Morocco, Fez, Morocco \\ ${ }^{3}$ Faculty of Medicine and Pharmacy, Sidi Mohamed Ben Abdellah University, Fez, Morocco \\ Email: ^harmouchi.hicham@gmail.com
}

How to cite this paper: Hicham, H., Sani, R., Ibrahim, I., Kassim, S., Layla, B., Fatimazahra, A., Marouane, L., Yassine, O. and Mohammed, S. (2018) Surgical Management of Pyothorax: A Series of 172 Cases. Open Journal of Thoracic Surgery, 8, 50-56. https://doi.org/10.4236/ojts.2018.82009

Received: May 10, 2018

Accepted: June 23, 2018

Published: June 27, 2018

Copyright $\odot 2018$ by authors and Scientific Research Publishing Inc. This work is licensed under the Creative Commons Attribution International License (CC BY 4.0).

http://creativecommons.org/licenses/by/4.0/

\begin{abstract}
Introduction: The pyothorax or thoracic empyema is defined by the presence between the two pleural layers of a purulent liquid, or a non-purulent liquid, but with bacteriological or biochemical characteristics testifying to a microbial invasion. The use of early medical treatment prevents the passage to pleural encystment, whose management remains surgical. Material and Methods: Our study was performed retrospectively, in the department of thoracic surgery of CHU Hassan II of Fez, between 2010 and 2016, involving 172 patients operated for pyothorax. Results: Among the 172 patients included in our study, there were 110 men and 67 women. The average age was 32.11 years old. The clinical picture was dominated by pleural syndrome in $56 \%$ of cases, signs of tuberculous impregnation in $44.1 \%$ of cases. Preoperative preparation with thoracic drainage, bi-antibiotic therapy and respiratory physiotherapy was performed in $90 \%$ of cases. The attack was right in $55.8 \%$ of cases and left in $44.1 \%$. A chest CT scan performed in all patients showed pachypleuritis in all cases. The tuberculous pyothorax accounted for $54 \%$ of cases, those by intra pleural rupture of a pulmonary hydatid cyst in $10.4 \%$, para-pneumonic origin in $5.2 \%$ and post-traumatic in $4.06 \%$. The origin was undetermined in $26.16 \%$. A conservative posterolateral thoracotomy was performed in all our patients. The pulmonary release was done through the extrapleural plane in $94 \%$ of cases, and pleuropulmonary decortication performed in all cases. Atypical resection was associated in $4.3 \%$ of cases and peri-cytectomy in $13.9 \%$ of cases. Operative follow-up was simple in $79.6 \%$ of cases. The main postoperative complications were a prolonged aerial leak in $16.7 \%$, atelectasis in $4.5 \%$, a wall infection in $6.5 \%$, a hemothorax refrained in $3.2 \%$ of cases. The average follow-up was 2.5 years. Conclusion : Tuberculosis remains the most common etiology of pyothorax in our setting. Early management plays an
\end{abstract}


important role in reducing the morbidity and mortality of this pathology. However, surgery remains the only effective treatment at the pleural encystment stage.

\section{Keywords}

Pyothorax, Pachypleuritis, Thoracic Drainage, Decortication, Surgery

\section{Introduction}

The pyothorax or thoracic empyema is the presence between the two pleural layers of a frankly purulent liquid, or a clear or sleazy liquid but containing a majority of altered neutrophils [1]. Early and well-managed medical management, through antibiotic therapy, thoracic drainage, and respiratory physiotherapy, avoids the transition to the formation of a pachypleuritis that may involve respiratory function prognosis and whose surgery alone can lead to healing. Tuberculosis remains the most common etiology, especially in TB endemic countries. The goal of the surgery is to free the lung from its fibrous gangue which encloses it and compresses it against the mediastinum. This surgery remains difficult and requires the competence of the thoracic surgeon [2]. Our goal is to bring our experience in the management of pyothorax.

\section{Material and Methods}

This was a retrospective study performed in the department of thoracic surgery of Hassan II CHU in Fes for 7 years (January 2010-December 2016). We collected 172 patients admitted and operated for pyothorax. Patients with postoperative pyothorax or pneumonectomy cavity were not included in this study. The data were collected on a pre-established operating file including demographic, clinical and paraclinical parameters, the surgical procedure performed as well as the post-operative follow-up.

\section{Results}

We collected 172 patients operated for pyothorax including 110 men and 67 women, with a sex ratio of 1.6. The average age was 32.11 years old. The history of pulmonary tuberculosis was found in $42.2 \%$ of cases, a notion of active smoking in $13 \%$ of cases, a contact with dogs in $8.7 \%$ of cases, and a notion of closed chest trauma in $4 \%$ of cases. The clinical picture was dominated by a pleural syndrome (chest pain, cough, dyspnoea) in $56 \%$ of cases, signs of tuberculous impregnation (night sweats, fever, deterioration of the general condition) in $44.1 \%$ of cases, general poor condition in $24.7 \%$ of cases, retractable chest deformity in $11.8 \%$ of cases, and hydatic vomit with membrane rejection in $5.8 \%$ of cases. All patients had a chest x-ray (Figure 1), showing enzymatic pleurisy in $44.5 \%$ of cases, a hydro-aera image in $30 \%$ of cases, non-encysted pleurisy in 
$17.2 \%$ of cases, and a white lung in $8.3 \%$ of cases. Thoracic CT, performed in all patients, revealed pachypleuritis in all cases, a pyothorax compartmentalized in $83.1 \%$ of cases, unpyothorax free cavity in $10.4 \%$ (Figure 2), and a broken hydatid cyst appearance in the pleura with a floating membrane appearance in $3 \%$ of cases. Preoperative preparation with thoracic drainage, bi-antibiotic therapy and respiratory physiotherapy was performed in $90 \%$ of cases. The right side was involved in $55.8 \%$ of cases, and the left side in $44.2 \%$. In the past, the epidemiology of pulmonary arteriosclerosis in patients with tuberculosis was found in 54\% of cases, secondary to an intra pleural rupture of a pulmonary hydatid cyst in $10.4 \%$, parapneumonic origin in $5.2 \%$ and post-traumatic in 4.06 The origin was indeterminate in $26.16 \%$ of cases (Table 1 ). Bronchoscopy bronchoscopy, made in $29 \%$ of cases, objectified a bronchopleural fistula in 3 patients, an inflammatory aspect in 15 patients, and a normal appearance in 11 patients.. Spirometry was performed in 17 patients, objectifying a restrictive syndrome in 3 patients. Thus, in the context of research for renal impairment, a proteinuria test of 24 hours was required in $69.7 \%$ of cases, returning positive in a single patient, following his diabetic nephropathy.

The approach was a conservative posterolateral thoracotomy of the dorsal muscle in all patients. Pulmonary release was achieved through an extrapleural plane in $94 \%$ of cases. Surgical exploration showed a pleural sac empty of pus in $79.4 \%$ of cases. The procedure consisted of pleuropulmonary decortication in all patients, associated with atypical resection in $4.3 \%$ of cases and perikystectomy in $13.9 \%$ of cases. Mean hospitalization and thoracic drainage times were 7 days and 11 days, respectively. A stay in the intensive care unit was necessary in $9.8 \%$ of cases (17 patients), including 3 patients for the management of septic shock occurring immediately postoperatively. Operative follow-up was simple in $79.6 \%$ of cases. The main postoperative complications were prolonged aerial leakage in $16.7 \%$, atelectasis in $4.5 \%$, wall infection in $6.5 \%$ and hemothorax redrained in $3.2 \%$ of cases (Table 2). The anatomopathological study of the operative specimen showed active tuberculosis in $50 \%$ of cases, and inflammatory changes for the rest of the patients. The mean follow-up in this study was 2.5 years and no death cases were noted, and complete lung re-expansion in $96 \%$ of cases.

Table 1. Etiologies of pyothorax.

\begin{tabular}{cc}
\hline Etiologies of pyothorax & number of patients (Percentage \%) \\
\hline $\begin{array}{c}\text { Pleuropulmonary tuberculosis } \\
\text { Hydatid cyst broken in the pleura }\end{array}$ & $93(54 \%)$ \\
Parapneumonic origin & $18(10.4 \%)$ \\
Posttraumatic origin & $7(5.2 \%)$ \\
Indeterminate origin & $45(26.16 \%)$
\end{tabular}


Table 2. Main postoperative complications.

\begin{tabular}{cc}
\hline Postoperative complications & number of patients (Percentage \%) \\
\hline Extended air leak & $29(16.7 \%)$ \\
Atelectasis & $8(4.5 \%)$ \\
Infection of the wall & $11(6.5 \%)$ \\
hemothorax & $5(3.2 \%)$ \\
Septic shock & $3(1.74 \%)$ \\
\hline
\end{tabular}

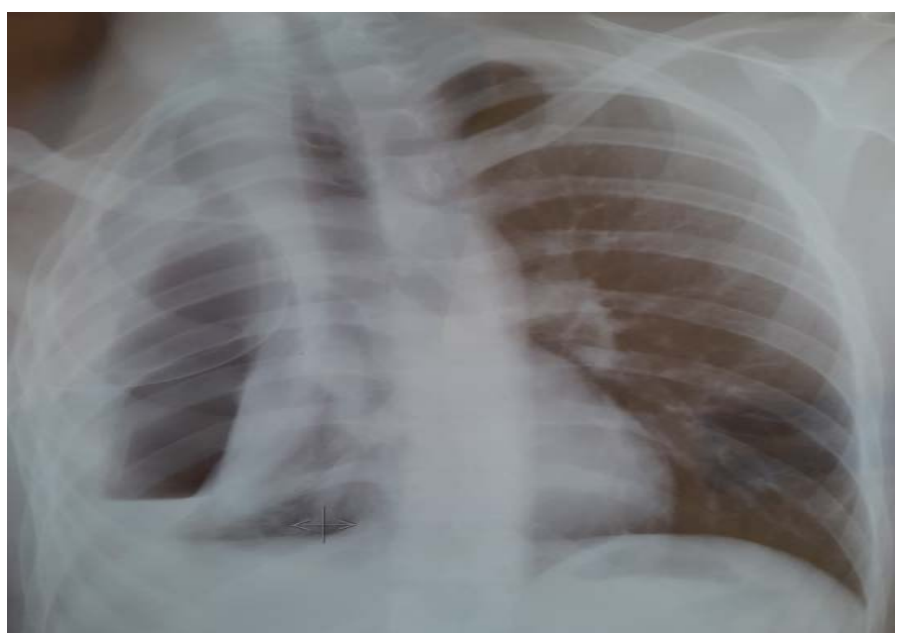

Figure 1. Chest $x$-ray showing a right pleural pocket, with apico-axillary opacity, and a retracted hemithorax (drain in place).

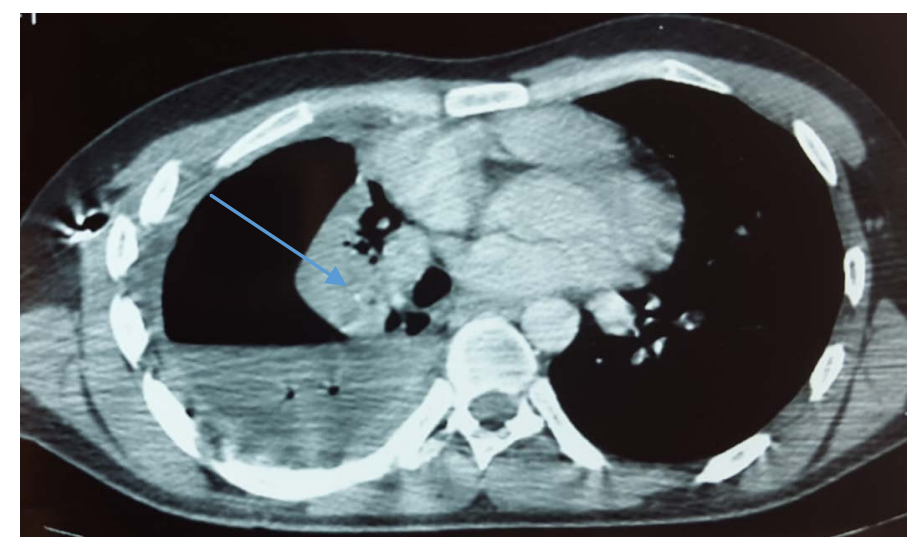

Figure 2. A mediastinal chest CT scan demonstrating drained pyo-pneumothorax with a retracted straight hemithorax and passive atelectasis of the underlying lung (arrow).

\section{Discussion}

The pyothorax, first described by Hippocrates 500 years before Jesus Christ, is an infectious and inflammatory pathology affecting the two pleural leaflets. It evolves in three phases: the diffusion phase where the effusion is free in the pleural cavity, and the pleura are still thin. In the fibrinopurulent phase, the fluid 
becomes thicker and more viscous with fibrin accumulation in the pleural space by inhibition of fibrinolytic activity [3]. In these first two phases medical treatment is effective and can lead to healing if it is early and well conducted. The third phase, called organization, corresponds to the proliferation of fibroblasts within the pleural cavity, with accumulation of fibrin and collagen responsible for a pachypleuritis preventing pulmonary re-expansion, causing restrictive sequelae [4], [5]. The evolution towards this last phase, whose treatment is only surgical, depends essentially on the initial management, the immunological status of the host, and the causative organism in pleurisy [6]. He can thus be at the origin of a table of serious infection sometimes with sepsis being able to put in play vital prognosis. In fact, 3 patients in this series had septic shock postoperatively requiring hospitalization in the intensive care unit. In our series, the young adult population was the most concerned with an average age of 32 years, hence a significant socio-economic impact. This finding has been made by several authors in the literature [7] [8] [9]. In our context, tuberculosis and hydatid cyst are the most common etiologies.. However, parapneumonic infection remains the most predominant cause in the other series [10]. Chest pain, cough and dyspnea remain the main clinical signs. In the chronic forms of thoracic deformities by retraction of the hemithorax can be observed. The clinical examination looks for a syndrome of fluid pleural effusion, a retracted hemithorax or an ENT or dental portal. The chest radiograph of face and profile, of systematic realization, made it possible to make the diagnosis, to determine the encysted or free nature of the pleural effusion. Thoracic CT was performed in all patients. It targets a pachypleuritis that engages the lung against the mediastinum and prevents any movement of the chest wall and diaphragm. It also helps to assess the quality of the underlying lung (passive atelectasis, pneumopathy, pulmonary abscess, tuberculous cavern, hydatid cyst). Bronchial fibroscopy is used to search for a local cause, causing pyothorax, or a broncho-pleural fistula [11]. One of the key steps in this management is preoperative preparation, based essentially on antibiotic therapy, thoracic drainage, respiratory physiotherapy and hypercaloric-hyperprotid diet. It can sterilize the pleural cavity, treat parenchymal infectious foci, thus avoiding per and post-operative sepsis. This medical preparation is all the more effective as it is performed in the early phases of purulent pleurisy, thus avoiding the progression to surgery. Decortication performed in all our patients is the basis of surgical treatment. It frees the lung from its fibrous gangue and restores the mobility of the wall and diaphragm [12]. It is a haemorrhagic gesture, which requires skill on the part of the thoracic surgeon. The approach to all our patients was a conservative posterolateral thoracotomy of the dorsal muscle. Sometimes resection of the rib is necessary especially for chronic pyothorax. An extrapleural detachment, made in all patients, is best performed on the finger, and should be done with caution not to damage some mediastinal structures [2]. In our series, atypical resection was performed in $4.3 \%$ of cases, indicating good preoperative preparation. Perkystectomy, sometimes with 
blindness of bronchial fistulas, was associated with decortication in $10.4 \%$ of cases in pyothorax of hydatid origin. All our patients had undergone postoperative monitoring, with a chest $\mathrm{x}$-ray every $48 \mathrm{~h}$. The average follow-up in our series was 2.5 years, and no deaths were recorded. Two patients had septic shock immediately postoperatively, requiring an average stay of 5 days in the intensive care unit. The follow-up was simple in $79.6 \%$ of cases. Prolonged bubbling was noted in $16.7 \%$ of patients treated by simple drainage. Poor analgesia, and consequently poor postoperative physiotherapy, increases the frequency of pneumopathies and atelectases [13]. The infections of the wall remain rather frequent after surgery of the pyothorax; they were found in $6.5 \%$ of the cases in our study, and managed by antibiotic therapy, and a daily change of the dressing. A postoperative hemothorax was found in $3.2 \%$ of our patients, requiring thoracic drainage, without surgical revision.

\section{Conclusion}

Pyothorax is the ultimate stage of evolution of purulent pleurisy. Pleuropulmonary tuberculosis and hydatid cyst remain the most common causes in our context. Although this study is retrospective, its interest is that it includes a large number of patients and highlights the role of decortication in the management of pyothorax with fewer postoperative complications, especially with good intraoperative pulmonary ventilation.

\section{Conflict of Interest}

The authors declare that they have no conflict of interest with this manuscript.

\section{References}

[1] Fantin, B. and Touaty, E. (1988) Pleuresies Purulentes. Encycl Med Chir, 6041, A1-15.

[2] Riquet, M. and Arab, M. (2004) Techniques of Decortication. Medical-Surgical Encyclopedia, 42, 462.

[3] Letheulle, J., Kerjouan, M., Bénézit, F., De Latour, B., Tattevin, P., Piau, C., Léna, H., Desrues, B., Le Tulzo, Y. and Jouneau, S. (2015) Parapneumonic Pleural Effusions: Epidemiology, Diagnosis, Classification and Management. Review of Respiratory Diseases, 344-357.

[4] Foulon, G., Debray, M.P. and Crestani, B. (2009) Purulent Pleurisy. In : Aubier, M., Crestani, B., Fournier, M. and Mal, H., Ees., Traité de Pneumologie, 2nd Edition, Médecine-Sciences Flammarion, 415-420.

[5] Light, R.W. (2006) Parapneumonic Effusions and Empyema. Proceedings of the American Thoracic Society, 3, 75-80. https://doi.org/10.1513/pats.200510-113JH

[6] Riquet, M. and Badia, A. (2003) Surgical Problems Posed by Purulent Pleurisy. In: Encycl Méd Chir, Surgical Techniques-Thorax, Elsevier SAS, Paris, 42-458.

[7] Thielen, S., Woimant, M., Poulet, C., Bentayeb, H., Lecuyer, E., Boutemy, M., et al. (2007) Management of Purulent Pleurisy: A Descriptive Study of 30 Patients. Summaries of Scientific Papers, 129. 
[8] Dagnra, A.Y., Awesso, B., Prince-David, M. and Tidjani, O. (2003) Nature and Antibiotic Sensitivity of Bacteria Isolated from Purulent Pleurisy in Lome (Togo). Medecine Et Maladies Infectieuses, 3, 327-330. https://doi.org/10.1016/S0399-077X(03)00201-4

[9] Fettal, N. and Taleb, A. (2011) Purulent Pleurisy: About 37 Cases. Revue des Maladies Respiratoires, 28, A66.

[10] Jebrak, G., Pointet, P. and Pichot, M.H. (1988) Purulent Pleurisy and Empyema. Presse Medicale, 27, 1924-1931.

[11] Davies, H.E., Davies, R.J.O. and Davies, C.W.H. (2010) Management of Pleural Infection in Adults: British Thoracic Society Pleural Disease Guideline 2010. Thorax 2010, 65, ii 41-53.

[12] Fantin, B. and Touaty, E. (1988) Purulent Pleurisy. Encycl Med Chir, Lung, 6041, A1-15.

[13] Lançon, J.P., Caillard, B., Coulon, C., Beaulieu, C. and Viard, H. (1986). Postoperative Complications of Pulmonary Surgery. Lyon Chirurgical, 82, 14-17. 